\title{
Perda de nutrientes via escoamento superficial no sistema plantio direto sob adubação mineral e orgânica
}

\author{
Nutrient losses by surface runoff in no-till system soil under mineral and organic fertilization
}

\section{Oromar João Bertol ${ }^{\mathrm{I}}$ Nivaldo Eduardo Rizzi ${ }^{\mathrm{II}}$ Emerson Fey ${ }^{\mathrm{III}}$ Maria do Carmo Lana ${ }^{\mathrm{III}}$}

\section{RESUMO}

\begin{abstract}
$O$ sistema de plantio direto (SPD) tem contribuído para melhorias nos solos agrícolas, dentre elas o aumento da concentração de nutrientes no solo. No entanto, tem se verificado que este aumento ocorre principalmente na camada superficial do solo, o que contribui para que ocorram perdas de nutrientes através de enxurradas, com danos econômicos e ambientais. Este trabalho avaliou a concentração total dos nutrientes potássio $(\mathrm{K})$, cálcio $(\mathrm{Ca})$, magnésio $(\mathrm{Mg})$ e cobre (Cu) e do $\mathrm{K}$ nas formas solúvel e particulado, na enxurrada ocasionada por chuvas simuladas com diferentes intensidades aplicadas sobre solo manejado por cinco anos sob SPD, após aplicação superficial de adubo orgânico ou mineral. Os adubos não afetaram a concentração dos nutrientes estudados, exceto na condição da chuva mais intensa e solo sob adubação orgânica, onde a enxurrada apresentou maior concentração de $K$ solúvel e $K$ total. Independente do tipo de adubo utilizado, a chuva de maior intensidade ocasionou maior concentração de todos os nutrientes e formas estudados, evidenciando os benefícios econômicos e ambientais da adoção de práticas de contenção da enxurrada, mesmo em áreas cultivadas sob SPD.
\end{abstract}

Palavras-chave: dejeto líquido de suínos, qualidade da água, erosão do solo, erosão em entressulcos.

\section{ABSTRACT}

The no-tillage system, has contributed to improvements in agricultural soils, among them the like increases in nutrient concentrations.of mineral elements in soil. However, it has been found that this increase concentration occurs mainly in the first few centimeters of the superficial top layer and so risks of nutrient losses by runoff, and associated of the soil, which concurs for losses of mineral elements through of runoff, with economic and environmental damages, are high. This study evaluated the total concentration, in runoff, of mineral elements potassium $(K)$, calcium $(\mathrm{Ca})$, magnesium $(\mathrm{Mg})$ and copper $(\mathrm{Cu})$ in total form and the soluble and particulate concentration form of $K$, in runoff caused to simulated rainfall of different intensitiesy applied over soil under no-till system and submitted to simulated after application of organic or mineral fertilizers in no-till soil. The fertilizers did not affect the concentration of mineral elements studied nutrients in runoff, except in condition of more high intense rainfall and soil under after organic manure application, which promoted runoff with showed the higher highest concentration of soluble and total $K$. Independent Irrespective of the type of fertilizers utilized type, the rainfall of higher intensity resulted in higher concentrations of all the mineral elements and forms studied, either in total, soluble or particulate form, evidencing the economic and environmental benefices, risks and suggesting that with the adoption of practices for runoff contention of runoff practices must be adopted, even in tilled croplands areas managed under no-till system.

Key words: liquid swine manure, losses of nutrients, water quality, soil erosion, interrill erosion.

\section{INTRODUÇÃO}

O sistema de plantio direto (SPD) favorece a concentração de nutrientes no solo, particularmente na superfície (McDOWELL \& SHARPLEY, 2001; CASSOL et al., 2002; SHARPLEY et al., 2001), pela

IÁreas de Meio Ambiente e Geoprocessamento, Instituto EMATER, Rua da Bandeira, 500, 80035-270, Curitiba, PR, Brasil. Email: oromar@emater.pr.gov.br. Autor para correspondência.

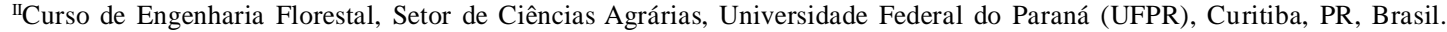

IIICentro de Ciências Agrícolas, Campus de Marechal Cândido Rondon, Universidade Estadual do Oeste do Paraná (UNIOESTE), Marechal Cândido Rondon, PR, Brasil. 
mínima mobilização desta camada (ISMAIL et al., 1994). Por outro lado, nesse sistema, a perda de água, que é o veículo para o transporte dos nutrientes, permanece elevada, fazendo com que as perdas na forma dissolvida sejam mais expressivas (EGHBALL\& GILLEY, 1999).

Além do sistema de manejo do solo, outros fatores, como o tipo de adubo utilizado e, principalmente, a forma de aplicação, exercem influência na distribuição de nutrientes no perfil. Os dejetos de animais têm sido cada vez mais usados na agricultura, como alternativa econômica de adubação das culturas. Estes materiais, no entanto, são distribuídos na superfície do solo e, por isso, têm favorecido a concentração de nutrientes nas camadas superiores do solo, comparativamente aos adubos de natureza mineral, que são incorporados na linha de semeadura (GINTING et al., 1998). Assim, o uso de adubos orgânicos cria um potencial para uma maior perda de nutrientes, uma vez que, estando na superfície do solo, estes são facilmente transportados pela enxurrada (SHARPLEY et al., 1991). Além disso, alguns dejetos são também fonte de metais pesados, dentre eles o cobre $(\mathrm{Cu})$, que em excesso, causa danos ao meio ambiente e à saúde humana. Este elemento, quando atinge níveis elevados no organismo, pode ocasionar vários problemas de saúde, dentre eles a doença de Wilson (SARGENTELLI et al., 1996).

A erosão hídrica, por ser o mais importante meio de transporte dos nutrientes das lavouras para os mananciais de água (GBUREK \& SHARPLEY, 1998), é um dos maiores causadores de contaminação de águas superficiais (PARRY, 1998). As duas principais formas de erosão hídrica são a erosão em entressulcos e a erosão em sulcos. Embora a perda de sedimentos na erosão em sulcos seja maior do que na erosão em entressulcos, esta é usualmente mais seletiva do que a primeira, não devendo ser desprezada como agente de poluição (PALIS et al., 1990), uma vez que transporta principalmente partículas coloidais de pequeno tamanho (MEYER et al., 1975). Estas partículas, constituídas basicamente de matéria orgânica, normalmente são altamente enriquecidas de nutrientes (BARROWS \& KILMER, 1963).

Dentro das estratégias de melhoria da qualidade das águas, a implantação de sistemas de terraceamento tem se apresentado como uma alternativa importante e viável. Os terraços são considerados como práticas conservacionistas complementares, que tem a função principal de controlar a erosão hídrica reduzindo o comprimento do declive, pelo seccionamento das encostas. Essas estruturas, ao reduzir o comprimento do declive, quebram a velocidade da enxurrada, acumulando-a no canal do terraço aumentando a infiltração da água e sua permanência no solo (PARANÁ, 1994).

O objetivo deste trabalho foi determinar a perda de nutrientes por erosão em entressulcos, em Latossolo manejado sob sistema de plantio direto, sob adubação orgânica e mineral. A finalidade foi obter informações para fundamentar a adoção de terraceamento para controle da enxurrada em lavouras manejadas nesse sistema e, conseqüentemente, reduzir a poluição difusa ocasionada pela erosão hídrica.

\section{MATERIAL E MÉTODOS}

O trabalho de campo foi realizado em outubro de 2003, em lavoura localizada no Município de Marechal Cândido Rondon, na Região Oeste do Estado do Paraná. A área experimental está localizada nas coordenadas UTM 194.290 e 7.283.460. O clima da região caracteriza-se por não apresentar estação seca, com verões quentes, cuja temperatura média do mês mais quente é de $22^{\circ} \mathrm{C}$, segundo Köepen, classificado como Cfa. O solo da área experimental é um Latossolo Vermelho eutroférrico (EMBRAPA, 2006), com declividade média de $0,045 \mathrm{~m} \mathrm{~m}^{-1}$, manejado nos últimos cinco anos sob sistema de plantio direto (SPD), com as seguintes propriedades físicas e químicas na camada de 0 a $0,2 \mathrm{~m}: 5,4$ de $\mathrm{pH} \mathrm{CaCl}_{2} ; 0 \mathrm{cmol}_{\mathrm{c}} \mathrm{kg}^{-1} \mathrm{Al}^{+3} ; 4,5 \mathrm{cmol}_{\mathrm{c}}$ $\mathrm{kg}^{-1} \mathrm{de} \mathrm{Ca}^{+2} ; 2,2 \mathrm{cmol}_{\mathrm{c}} \mathrm{kg}^{-1}$ de $\mathrm{Mg}^{+2} ; 0,34 \mathrm{cmol}_{\mathrm{c}} \mathrm{kg}^{-1} \mathrm{de}$ $\mathrm{K}^{+} ; 54,1 \mathrm{mg} \mathrm{kg}^{-1}$ de P Mehlich I; $729 \mathrm{~g} \mathrm{~kg}^{-1}$ de argila; $188 \mathrm{~g} \mathrm{~kg}^{-1}$ de silte; $83 \mathrm{~g} \mathrm{~kg}^{-1}$ de areia; $1,29 \mathrm{~kg} \mathrm{dm}^{-3}$ de densidade do solo; $2,74 \mathrm{~kg} \mathrm{dm}^{-3}$ de densidade de partículas; $0,41 \mathrm{dm}^{3} \mathrm{dm}^{-3} \mathrm{de}$ microporos; $0,11 \mathrm{dm}^{3} \mathrm{dm}^{-3}$ de macroporos e $0,52 \mathrm{dm}^{3} \mathrm{dm}^{-3}$ de porosidade total. A densidade do solo e de partículas foram determinadas, respectivamente, pelos métodos do anel e do balão volumétrico (EMBRAPA, 1997).

Antes da implantação dos tratamentos foi executada uma operação de semeadura, com uma máquina semeadora contendo sulcadores de cinzel, distanciados de $0,4 \mathrm{~m}$ entre si. Esta operação foi realizada no sentido da maior declividade do terreno, por representar uma condição que geralmente é praticada em lavouras sem sistema de terraceamento agrícola implantado, bem como por criar uma condição crítica de ocorrência de escoamento superficial e de transporte de solo por erosão hídrica.

As parcelas experimentais, com dimensão de $1 \mathrm{~m} \times 1 \mathrm{~m}$ cada, foram isolada com chapas metálicas, encaixando-se uma calha coletora autolimpante na extremidade inferior da parcela, para coletar a enxurrada gerada no interior da mesma. Para armazenar essa enxurrada, foi aberta uma trincheira em frente à calha, com dimensões e profundidade suficientes para 
comportar um reservatório com capacidade de armazenamento para 25 litros, o qual foi conectado à calha coletora por meio de uma mangueira.

Os tratamentos de adubação utilizados foram: adubação orgânica $(\mathrm{AO})$, na forma de dejeto líquido de suínos (DLS) aplicado na quantidade 6L parcela ${ }^{-1}\left(60 \mathrm{~m}^{3} \mathrm{ha}^{-1}\right)$, correspondente a $48,6 \mathrm{~kg} \mathrm{ha}^{-1} \mathrm{de}$ $\mathrm{K} ; 47,4 \mathrm{~kg} \mathrm{ha}^{-1} \mathrm{de} \mathrm{Ca}, 31,8 \mathrm{~kg} \mathrm{ha}^{-1} \mathrm{de} \mathrm{Mg}, 5,0 \mathrm{~kg} \mathrm{ha}^{-1} \mathrm{de}$ $\mathrm{Cu}$ e $4,0 \mathrm{~kg} \mathrm{ha}^{-1}$ de $\mathrm{Zn}$, conforme análise realizada no Laboratório de Fertilizantes e Calcários do TECPAR (BRASIL, 1982); adubação mineral (AM), composta de $\mathrm{K}$ na forma de cloreto de potássio, aplicado na quantidade de $20 \mathrm{~g} \mathrm{parcela}{ }^{-1}\left(200 \mathrm{~kg} \mathrm{ha}^{-1}\right)$, correspondendo a $30,4 \mathrm{~kg} \mathrm{ha}^{-1}$ de $\mathrm{K}$, conforme análise do adubo realizada no Laboratório de Fertilizantes e Calcários do TECPAR (BRASIL, 1982); e testemunha, sem adubação (T). Os tratamentos foram aplicados em parcelas distribuídas aleatoriamente sob a área de ação do simulador de chuva, em quatro repetições.

As chuvas, com o uso de simulador de chuva de braços rotativos (SWANSON, 1975), foram iniciadas dezesseis horas após a aplicação dos tratamentos. Inicialmente foi aplicada uma chuva para umedecimento do solo, com intensidade média de $55 \mathrm{~mm}$ $\mathrm{h}^{-1}$ e duração de 38 minutos. Trinta minutos após o término dessa chuva, foi aplicada uma série de três chuvas simuladas em intervalos de 30 minutos entre si, todas com duração de 20 minutos, sendo a primeira chuva com intensidade de $70 \mathrm{~mm} \mathrm{~h}^{-1}(\mathrm{C} 1)$; a segunda chuva com intensidade de $60 \mathrm{~mm} \mathrm{~h}^{-1}(\mathrm{C} 2)$ e, a terceira chuva com intensidade de $120 \mathrm{~mm} \mathrm{~h}^{-1}(\mathrm{C} 3)$.

Após a aplicação de cada chuva (C1, C2 e C3), os reservatórios contendo as enxurradas das parcelas foram conduzidos para o laboratório, homogeneizados e coletadas amostras de $200 \mathrm{~mL}$ e $500 \mathrm{~mL}$ de volume. As amostras com volume de $200 \mathrm{ml}$ foram centrifugadas, sendo o sobrenadante filtrado em filtro de $0,45 \mathrm{~mm}$ de abertura de malha e determinada a concentração de K solúvel (KS) na enxurrada, em espectrofotômetro de absorção atômica. As amostra de enxurrada com volume de $500 \mathrm{ml}$ foram utilizadas para determinar as concentrações de sedimentos (BERTOL et al., 2007), K total (KT), Ca total (CaT), Mg total $(\mathrm{MgT})$ e $\mathrm{Cu}$ total $(\mathrm{CuT})$. A concentração de sedimentos na enxurrada foi determinada submetendose as amostras à secagem em estufa a $65^{\circ} \mathrm{C}$ até a eliminação da água e, a seguir, a $105^{\circ} \mathrm{C}$ por 24 horas, sendo a massa de sedimento determinada em balança de precisão de quatro dígitos. As concentrações de $\mathrm{KT}, \mathrm{CaT}, \mathrm{MgT}$ e CuT na enxurrada foram determinadas submetendo os sedimentos à digestão total (MARTINEZ, 1970) e análise em espectrofotômetro de absorção atômica. O K particulado (KP) foi obtido subtraindo-se o KS do KT.
O delineamento experimental foi o inteiramente aleatorizado, com distribuição fatorial dos tratamentos de adubação orgânica (AO), adubação mineral (AM) e sem adubo, testemunha (T) versus chuva $1(\mathrm{C} 1)$, chuva $2(\mathrm{C} 2)$ e chuva $3(\mathrm{C} 3)$. As comparações múltiplas foram realizadas por contrastes ortogonais do teste F.

\section{RESULTADOS E DISCUSSÃO}

Os adubos aplicados no solo proporcionaram aumento na concentração de K solúvel (KS) na enxurrada (Tabela 1). O maior aumento foi proporcionado pelo dejeto líquido de suínos (DLS) na última chuva (C3), com uma concentração em torno de duas vezes à do solo não adubado (T). Os resultados podem ser atribuídos à baixa reatividade do $\mathrm{K}$ com o solo (BLAYA \& NAVARRO, 1984), associado à condição de solubilidade do elemento. A intensidade da chuva influenciou significativamente na concentração de KS na enxurrada (Tabela 1), tendo a chuva mais intensa (C3) apresentado os maiores valores. Estes resultados são ainda mais expressivos se considerados o fato da chuva de maior intensidade ter sido a última da série e que há propensão do $\mathrm{K}$ à solubilização (CERETTA et al. 2003), o que permitiria esperar que as maiores concentrações ocorressem nas enxurradas das primeiras chuvas $(\mathrm{C} 1 \mathrm{e} \mathrm{C} 2)$.

A concentração de K particulado (KP) nos sedimentos totais da enxurrada não foi afetada pelo tipo de adubo aplicado no solo (Tabela 1), o que pode ser atribuído a baixa afinidade do $\mathrm{K}$ com os constituintes do solo (SPARKS, 1995). No entanto, a intensidade das chuvas influenciou significativamente os resultados, tendo a chuva mais intensa (C3) ocasionado enxurrada com concentração de $\mathrm{KP}$ em torno de $120 \%$ mais elevada do que as demais chuvas, enquanto que a diferença de intensidade entre as chuvas foi de $60 \%$. Os resultados podem ser creditados ao efeito combinado da maior concentração de sedimentos na enxurrada da chuva C3 (BERTOL et al., 2007) com o fato de que o DLS é absorvido preferencialmente pelos grandes agregados do solo (BHATNAGAR et al., 1985), provavelmente em maior proporção na enxurrada da chuva mais intensa (C3).

A aplicação ou não de adubo, não influenciou na concentração de K total (KT) na enxurrada (Tabela 1), embora os resultados indiquem valores mais elevados de KT na enxurrada proveniente do solo adubado com DLS, principalmente na chuva C3. É provável que a ausência de diferença estatística esteja relacionada com a variabilidade dos dados, também constatado por CASSOL et al. (2002), que na 
Tabela 1 - Contraste de médias da concentração de K solúvel (KS), K particulado (KP) e K total (KT) na enxurrada de três chuvas simuladas (C1, C2, C3), aplicadas nos tratamentos de adubação orgânica (AO), adubação mineral (AM) e controle (T).

\begin{tabular}{|c|c|c|c|c|c|}
\hline \multirow{2}{*}{ Chuva } & \multicolumn{3}{|c|}{ 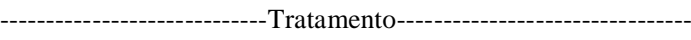 } & \multicolumn{2}{|c|}{ - } \\
\hline & $\mathrm{AO}$ & $\mathrm{AM}$ & $\mathrm{T}$ & $\mathrm{T}$ vs $\mathrm{AO}, \mathrm{AM}$ & $\mathrm{AO}$ vs $\mathrm{AM}$ \\
\hline & -------------- & $\mathrm{KS}, \mathrm{mg} \mathrm{L}^{-1}$ & 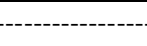 & & \\
\hline $\mathrm{C} 1$ & $5,38 \pm 1,03$ & $4,84 \pm 0,75$ & $2,85 \pm 0,20$ & 0,036 & 0,627 \\
\hline $\mathrm{C} 2$ & $5,03 \pm 0,64$ & $5,03 \pm 0,90$ & $2,66 \pm 0,16$ & 0,015 & 1,000 \\
\hline $\mathrm{C} 3$ & $8,25 \pm 0,89$ & $4,66 \pm 1,27$ & $4,68 \pm 0,61$ & 0,166 & 0,027 \\
\hline Contrastes & & $\operatorname{Pr}>\mathrm{F}$ & & & \\
\hline $\mathrm{C} 1$ vs $\mathrm{C} 2, \mathrm{C} 3$ & 0,266 & 0,998 & 0,114 & & \\
\hline $\mathrm{C} 2$ vs $\mathrm{C} 3$ & 0,028 & 0,798 & 0,005 & & \\
\hline & - & $\mathrm{mg} \mathrm{L}^{-1}$ de $\mathrm{KP}$ & ------- & & \\
\hline $\mathrm{C} 1$ & $5,70 \pm 1,22$ & $4,24 \pm 0,97$ & $5,18 \pm 0,73$ & 0,866 & 0,325 \\
\hline $\mathrm{C} 2$ & $5,06 \pm 0,33$ & $4,65 \pm 0,44$ & $5,32 \pm 0,62$ & 0,446 & 0,562 \\
\hline $\mathrm{C} 3$ & $12,66 \pm 1,46$ & $12,80 \pm 2,17$ & $9,76 \pm 1,96$ & 0,232 & 0,958 \\
\hline Contrastes & & $\operatorname{Pr}>\mathrm{F}$ & & & \\
\hline $\mathrm{C} 1$ vs $\mathrm{C} 2, \mathrm{C} 3$ & 0,046 & 0,028 & 0,160 & & \\
\hline $\mathrm{C} 2$ vs $\mathrm{C} 3$ & 0,001 & 0,003 & 0,034 & & \\
\hline & - & $\mathrm{mg} \mathrm{L}^{-1}$ de KT & - & 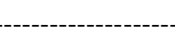 & - \\
\hline $\mathrm{C} 1$ & $11,08 \pm 2,24$ & $9,08 \pm 1,55$ & $8,03 \pm 0,86$ & 0,337 & 0,415 \\
\hline $\mathrm{C} 2$ & $10,09 \pm 0,77$ & $9,68 \pm 1,21$ & $7,98 \pm 0,74$ & 0,130 & 0,764 \\
\hline C3 & $20,90 \pm 1,67$ & $17,46 \pm 2,15$ & $14,44 \pm 1,39$ & 0,056 & 0,202 \\
\hline Contraste & & $\operatorname{Pr}>\mathrm{F}$ & & & \\
\hline $\mathrm{C} 1$ vs $\mathrm{C} 2, \mathrm{C} 3$ & 0,060 & 0,058 & 0,033 & & \\
\hline $\mathrm{C} 2$ vs $\mathrm{C} 3$ & 0,001 & 0,010 & 0,002 & & \\
\hline
\end{tabular}

$\operatorname{Pr}>\mathrm{F}=\mathrm{P}$-valor da estatística $\mathrm{F}$ para contrastes ortogonais;

chuva $\mathrm{C} 3$, apresentaram CV\% de 8, 12 e 10 para $\mathrm{AO}$, MS e T respectivamente. No entanto, maiores concentrações de KT na enxurrada poderiam ser esperadas do tratamento AO, especialmente nas chuvas iniciais, em função da maior quantidade de $\mathrm{K}$ aplicada na forma de dejeto $\left(48,6 \mathrm{~kg} \mathrm{ha}^{-1}\right.$, em torno de $60 \%$ superior à quantidade aplicada no tratamento com fertilizante mineral, de $30,4 \mathrm{~kg} \mathrm{ha}^{-1}$ ) e de que a quase totalidade do K presente no DLS está na forma solúvel (CERETTA et al., 2003), portanto mais suscetível ao transporte principalmente pela enxurrada das primeiras chuvas. Na comparação entre chuvas, os resultados mostram maior concentração de KT na enxurrada da chuva $\mathrm{C} 3$, em todos os tratamentos, mas com valores mais elevados no tratamento AO. À semelhança do $\mathrm{KP}$, isto pode ser creditado ao fato de que o DLS é absorvido preferencialmente pelos grandes agregados do solo (BHATNAGAR et al., 1985), provavelmente presente em maior proporção na enxurrada da chuva C3, em razão da maior intensidade desta chuva que, associada à maior umidade antecedente, provocou a desagregação e transporte desses.

A concentração de todas as formas de $\mathrm{K}$ estudadas, no tratamento sem adubação, pode ser considerada elevada. Os resultados certamente se devem à lixiviação do $\mathrm{K}$ dos restos de culturas que se acumularam sobre o solo (GIACOMINI et al., 2003) e ao SPD empregado na área experimental antes da aplicação dos tratamentos, o que favoreceu o enriquecimento da superfície do solo com nutrientes.

As concentrações de Ca total (CaT) e de Mg total (MgT) na enxurrada não foram influenciadas pelos tratamentos (Tabela 2) embora tenha havido aplicação destes elementos no tratamento $\mathrm{AO}$ através do DLS. Os resultados são explicados pelo efeito combinado da baixa concentração de $\mathrm{Ca}$ e $\mathrm{Mg}$ no dejeto $\left(0,79 \mathrm{e} 0,53 \mathrm{~kg} \mathrm{~m}^{-3}\right.$, respectivamente), também constatado por CERETTA et al. (2003), com as condições originais da camada de solo de 0 a $0,25 \mathrm{~m}$ que era de altos teores de $\mathrm{Ca}$ e $\mathrm{Mg}\left(4,5 \mathrm{cmol}_{\mathrm{c}} \mathrm{kg}^{-1} \mathrm{de} \mathrm{Ca}^{2+}\right.$ e 2,2 $\mathrm{cmol}_{\mathrm{c}} \mathrm{kg}^{-1} \mathrm{de}$ $\left.\mathrm{Mg}^{2+}\right)$.

Em todos os tratamentos, a enxurrada da chuva C3 apresentou concentração mais elevada tanto de CaT como de $\mathrm{MgT}$ em relação as chuvas $\mathrm{C} 1$ e C2. Os resultados indicam um efeito da intensidade da chuva na remoção das bases do solo, uma vez que a concentração de KT na enxurrada (Tabela 1), também foi maior na chuva $\mathrm{C} 3$ em relação às demais. 
Tabela 2 - Contraste de médias da concentração de Ca total (CaT), Mg total (MgT) e Cu total (CuT), na enxurrada de três chuvas simuladas (C1, C2, C3) aplicadas nos tratamentos de adubação orgânica (AO), adubação mineral (AM) e controle (T).

\begin{tabular}{|c|c|c|c|c|c|}
\hline \multirow{2}{*}{ Chuva } & \multicolumn{3}{|c|}{ 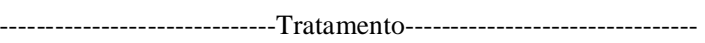 } & \multicolumn{2}{|c|}{ 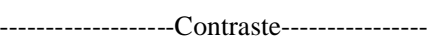 } \\
\hline & $\mathrm{AO}$ & $\mathrm{AM}$ & $\mathrm{T}$ & $\mathrm{T}$ vs $\mathrm{AO}, \mathrm{AM}$ & $\mathrm{AO}$ vs $\mathrm{AM}$ \\
\hline & \multicolumn{5}{|c|}{ - } \\
\hline $\mathrm{C} 1$ & $7,87 \pm 1,15$ & $5,87 \pm 0,80$ & $6,30 \pm 0,47$ & 0,601 & 0,132 \\
\hline $\mathrm{C} 2$ & $6,46 \pm 0,43$ & $6,47 \pm 0,25$ & $6,95 \pm 0,44$ & 0,325 & 0,987 \\
\hline $\mathrm{C} 3$ & $16,80 \pm 1,76$ & $17,09 \pm 3,18$ & $12,73 \pm 2,61$ & 0,216 & 0,939 \\
\hline Contraste & & $\operatorname{Pr}>\mathrm{F}$ & & & \\
\hline $\mathrm{C} 1$ vs $\mathrm{C} 2, \mathrm{C} 3$ & 0,035 & 0,032 & 0,096 & & \\
\hline $\mathrm{C} 2$ vs $\mathrm{C} 3$ & 0,0002 & 0,003 & 0,027 & & \\
\hline & & $\mathrm{L}^{-1} \mathrm{de} \mathrm{MgT}$ & - & & \\
\hline $\mathrm{C} 1$ & $2,75 \pm 0,31$ & $2,03 \pm 0,51$ & $2,09 \pm 0,27$ & 0,530 & 0,212 \\
\hline $\mathrm{C} 2$ & $3,24 \pm 0,42$ & $2,49 \pm 0,20$ & $2,58 \pm 0,21$ & 0,449 & 0,106 \\
\hline $\mathrm{C} 3$ & $6,85 \pm 0,70$ & $6,67 \pm 0,60$ & $5,45 \pm 1,21$ & 0,252 & 0,890 \\
\hline Contraste & & $\operatorname{Pr}>F$ & & & \\
\hline $\mathrm{C} 1$ vs $\mathrm{C} 2, \mathrm{C} 3$ & 0,005 & 0,002 & 0,059 & & \\
\hline $\mathrm{C} 2$ vs $\mathrm{C} 3$ & 0,0007 & 0,0001 & 0,021 & & \\
\hline & 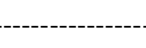 & $n g \mathrm{~L}^{-1} \mathrm{CuT}-$ & 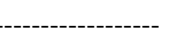 & ---------- & ----.-. \\
\hline $\mathrm{C} 1$ & $0,53 \pm 0,13$ & $0,06 \pm 0,01$ & $0,20 \pm 0,03$ & 0,322 & 0,002 \\
\hline $\mathrm{C} 2$ & $0,39 \pm 0,03$ & $0,28 \pm 0,02$ & $0,34 \pm 0,04$ & 0,960 & 0,039 \\
\hline $\mathrm{C} 3$ & $1,10 \pm 0,20$ & $0,98 \pm 0,23$ & $0,64 \pm 0,15$ & 0,134 & 0,678 \\
\hline Contraste & & $\operatorname{Pr}>\mathrm{F}$ & & & \\
\hline $\mathrm{C} 1$ vs $\mathrm{C} 2, \mathrm{C} 3$ & 0,242 & 0,008 & 0,027 & & \\
\hline $\mathrm{C} 2$ vs $\mathrm{C} 3$ & 0,006 & 0,005 & 0,040 & & \\
\hline
\end{tabular}

$\mathrm{Pr}>\mathrm{F}=\mathrm{P}-\mathrm{valor}$ da estatística F para contrastes ortogonais.

Os resultados se devem à alta reatividade do Ca e do Mg com o solo (BLAYA\& NAVARRO, 1984), associado à maior intensidade desta chuva, conferindo à enxurrada que se formou, uma maior capacidade de desagregação de partículas de solo e uma maior capacidade de transporte das partículas individualizadas. Outro aspecto a ser considerado é a composição dos sedimentos transportados, uma vez que, grande parte das partículas provavelmente se constituía de frações orgânicas acumuladas no SPD, as quais possuem uma elevada capacidade de reter $\mathrm{Ca}$ e Mg (MENDONÇA \& ROWELL, 1994).

O tipo de adubo utilizado influenciou a concentração de $\mathrm{Cu}$ total (CuT) na enxurrada, tendo o DLS proporcionado concentrações mais elevadas do que o adubo mineral, exceto na chuva $\mathrm{C} 3$ (Tabela 2). Os resultados são decorrentes da presença de elevada concentração deste elemento no DLS, em função da absorção parcial, pelo trato digestivo dos suínos, do $\mathrm{Cu}$ fornecido aos animais através da ração. A intensidade da chuva influenciou na concentração de Cu na enxurrada, uma vez que os valores mais elevados desta variável foram observados na enxurrada da chuva mais intensa (C3), à semelhança do que ocorreu com os demais nutrientes estudados. Os resultados podem ser explicados pela maior concentração de sedimentos na enxurrada desta chuva (BERTOL et al., 2007) e pela alta reatividade do $\mathrm{Cu}$ com o solo (GOMES et al., 2001). Isto mostra que a taxa de transferência deste elemento para a enxurrada depende da capacidade desta em transportar sedimentos. Enxurradas de maior volume possuem maior capacidade de transporte de sedimentos, em razão de sua maior energia de transporte (BERTOLet al., 2007).

Os resultados obtidos neste trabalho mostram, no que diz respeito às concentrações dos nutrientes estudados na enxurrada, que os valores se elevaram com a intensidade da chuva e com a concentração dos sedimentos na enxurrada para todos os nutrientes estudados. Evidencia-se, assim, a necessidade de conter a enxurrada na lavoura, mesmo sob o SPD. A contenção da enxurrada por meio de terraceamento bem dimensionado, por exemplo, permite que o efeito depurador do solo diminua a carga iônica da enxurrada (LARSON \& PIERCE, 1994; FONTES et al., 2001; PIOVESAN et al., 2009, BERTOL, 2005), jáque a água tenderá a se infiltrar no solo na região dos canais dos terraços. Além disso, os terraços controlam a 
dinâmica da enxurrada no que se refere às perdas de água e solo, repercutindo positivamente para os aspectos econômico e ambiental das propriedades rurais e, por conseqüência, da sociedade, uma vez que, em assim fazendo, serão evitadas tanto a perda de nutrientes das lavouras quanto à conseqüente degradação dos recursos naturais água e solo (BERTOL, et al., 2007).

\section{CONCLUSÃO}

O tipo de adubo utilizado, de fonte orgânica ou mineral, não influenciou a concentração dos nutrientes e formas destes (K solúvel, particulado e total e $\mathrm{Ca}, \mathrm{Mg}$ e $\mathrm{Cu}$ total) na enxurrada de erosão em entressulcos, tendo a chuva mais intensa, no entanto, proporcionado maiores concentrações. Os resultados obtidos neste estudo evidenciam a necessidade de contenção da enxurrada, mesmo em áreas manejadas sob o sistema plantio direto, o que pode ser obtido por meio da implantação de sistema de terraceamento corretamente dimensionado.

\section{REFERÊNCIAS}

BARROWS, S.G.; KILMER. V.J. Plant nutrient losses from soils by water erosion. Advance in Agronomy, v.15, p.303-316, 1963.

BERTOL, O.J. Contaminação da água de escoamento superficial e da água percolada pelo efeito de adubação mineral e adubação orgânica em sistema de semeadura direta. 2005. 209f. Tese (Doutorado em Ciências Florestais) - Setor de Ciências Agrárias, Universidade Federal do Paraná, PR. Disponível em: <http://dspace.c3sl.ufpr.br/dspace/bitstream/ 1884/2216/1/Tese_Oromar_Final.pdf >. Acesso em: 17 de jul. de 2010 .

BERTOL, O.J. et al. Perdas de solo e água e qualidade do escoamento Superficial associadas à erosão entre sulcos em área cultivada sob semeadura direta e submetida Às adubações mineral e orgânica. Revista Brasileira de Ciência do Solo, v.31, p.781-792, 2007. Disponível em: <http://www.scielo.br/ $\mathrm{scielo}$.php? script=sci_arttext \& pid=S 0100 $06832007000400018 \& \operatorname{lng}=$ pt\&nrm=iso $>$. Acesso em: $14 \mathrm{de}$ jan. de 2011. doi: 10.1590/S0100-06832007000400018.

BHATNAGAR, V.K. et al. Reaction of fertilizer and liquid manure phosphorus with soil aggregates and sediment phosphorus enrichment. Journal of Environmental Quality, v.14, p.246-251, 1985.

BLAYA, S.N.; NAVARRO, G.G. Temas de química agrícola: el suelo e los elementos quimicos esenciales para la vida vegetal. Leon: Academia, 1984. 601p.

BRASIL - Ministério da Agricultura. Métodos padrões oficiais para a análise de Fertilizantes. Laboratório de Referência Vegetal. Portaria SNAD n.31. Brasília, 1982.

CASSOL, E.A. et al. Perdas de nutrientes por erosão em diferentes métodos de melhoramento de pastagem nativa no
Rio Grande do Sul. Revista Brasileira de Ciência do Solo, v.26, p.705-712, 2002.

CERETTA, C.A. et al. Características químicas de solo sob aplicação de esterco líquido de suínos em pastagem natural. Pesquisa agropecuária brasileira, v.38, p.729-735, 2003.

EGHBALL, B.; GILLEY, J.E. Phosphorus and nitrogen in runoff following beef cattle manure or compost application to no-till and tilled soil. Journal of Environmental Quality, v.28, p.1201-1210, 1999.

EMBRAPA. Centro Nacional de Pesquisa de Solos-CNPS. Manual de métodos de análise de solo. 2.ed. Rio de Janeiro, 1997. 212p.

EMBRAPA. Centro Nacional de Pesquisa de Solos-CNPS. Sistema brasileiro de classificação de solos. 2.ed. Rio de Janeiro, 2006. 306p

FONTES, M.P.F. et al. Eletroquímica das partículas coloidais e sua relação com a mineralogia de solos altamente intemperizados. Scientia Agricola, v.58, p.627-646, 2001. Disponível em: <http://www.scielo.br/scielo.php?script=sci_arttext\&pid=S0103$90162001000300029 \& \operatorname{lng}=p t \& n r m=i s o \& t \operatorname{lng}=p t>$. Acesso em: 14 de jan. de 2011. doi: 10.1590/S0103-90162001000300029

GBUREK, W.J.; SHARPLEY, A.L. Hydrologic controls on phosphorus loss from upland agricultural watersheds. Journal of Environmental Quality, v.27, p.267-277, 1998.

GIACOMINI, S.J. et al. Liberação de fósforo e potássio durante a decomposição de resíduos culturais em plantio direto. Pesquisa Agropecuária Brasileira, v.38, p.1097-1104, 2003.

GINTING, D. et al. Interaction between manure and tillage system on phosphorus uptake and runoff losses. Journal of Environmental Quality, v.27, p.1403-1410, 1998.

GOMES, P.C. et al. Selectivity sequence and competitive adsorption of heavy metals by Brazilian soils. Soil Science Society of America Journal, v.65, p.1115-1121, 2001.

ISMAIL, I. et al. Long-term no-tillage effects on soil properties and continuous corn yields. Soil Science Society of America Journal, v.58, p.193-198, 1994.

LARSON, W.E.; PIERCE, F.J. The dynamics of soil quality as a measure of sustainable management. In: DORAN, J.W. et al. (Eds). Defining soil quality for a sustainable environment. Madison, SSSA, 1994. p.37-51. (Special, 35).

MARTINEZ, J.B. Analisis quimico de suelos. Barcelona: Omega, 1970. 662p.

McDOWELL, L.L.; SHARPLEY, A.N. Phosphorus losses in subsurface flow before and after manure application to intensively farmed land. Science of the Total Environment, v.278, p.113$125,2001$.

MENDONÇA, E.S.; ROWEL, D.L. Dinâmica do alumínio e de diferentes frações orgânicas de um Latossolo argiloso sob cerrado e soja. Revista Brasileira de Ciência do Solo, v.18, p.295303, 1994.

MEYER, L.D. et al. Source of soil eroded by water from upland slopes. In: ESTADOS UNIDOS. Department of Agriculture. 
Agriculture Research Service. Present and prospective technology for predicting sediment yields and sources. Washington: USDA-Agricultural Research, 1975. p.177-189.

PALIS, R.G. et al. Soil erosion processes and nutrient loss. I, The interpretation of enrichment ratio and nitrogen loss in Runoff sediment. Australian Journal of Soil Research, v.28, p.623-639, 1990 .

PARANÁ. Secretaria de Estado da Agricultura e do Abastecimento - SEAB. Embasamento técnico do subprograma de manejo e conservação do solo. 2.ed. CuritibaCidade, 1994. 306p.

PARRY, R. Agricultural phosphorus and water quality: a U.S. environmental protection agency perspective. Journal of Environmental Quality, v.27, p.258-261, 1998.

PIOVESAN, R.P et al. Perdas de nutrientes via subsuperfície em colunas de solo sob fertilização mineral e orgânica. Revista Brasileira de Ciência do Solo, v.33, p.757-766, 2009. Disponível em: <http:/ $/$ www.scielo.br/scielo.php?script=sci_arttext\&pid=S0100- 06832009000400002\&lng=pt\&nrm=iso $>$. Acesso em: 14 de jan. de 2011. doi: $10.1590 / \mathrm{S} 0100-06832009000400002$

SARGENTELLI, V. et al. Aspectos do metabolismo do cobre no homem. Química Nova, v.3, p.290-293, 1996.

SHARPLEY, A.N. et al. The meansurement of bioavailable phosphorus in agricultural runoff. Journal of Environmental Quality, v.20, p.235-238, 1991.

SHARPLEY, A.N. et al. Phosphorus loss from land to water: integrating agricultural and environmental management. Plant and Soil, v.237, p.287-307, 2001.

SPARKS, D.L. Environmental soil chemistry. San Diego: Academic, 1995. 267p.

SWANSON, N.P. Suggestions for the use of the rotatingboom field plot rainfall simulator to obtain data for application of the soil loss equation. Paraná: FAO/ University of Nebraska, 1975. 58p. (Relatório de consultoria). 